\title{
ON THE INTERPRETATION OF THE RELATIVE INTENSITIES
}

\section{OF THE SOLAR XUV LINES OF LITHIUM-LIKE IONS}

\author{
D. R. FLOWER \\ Observatoire de Paris, 92 - Meudon, France
}

The relative intensities of the $2 s^{-} 2 p$ and $2 l-3 l^{\prime}\left(l=0, l^{\prime}=1 ; l=1, l^{\prime}=0,2\right)$ solar XUV lines of ions in the lithium isoelectronic sequence are sensitive to electron temperature because of the large energy separation of the $2 p$ and $3 l^{\prime}$ levels. Recent observations of these lines for three members of the sequence, $\mathrm{O}^{+5}, \mathrm{Ne}^{+7}$ and $\mathrm{Mg}^{+9}$ (Heroux and Cohen, 1971), consequently provide a potentially powerful means of studying the temperature structure of the upper chromosphere-corona transition region. These observations have been examined in the light of recent calculations of the electron excitation cross-sections for the relevant transitions (Flower, 1971). It is found that the observed intensity ratios are systematically greater than values calculated assuming that all the lines of a given ion are produced at essentially the same temperature, namely, the temperature for which the ionization equilibrium calculations of Jordan (1969) predict that emission in the lines is most highly favou red. Part of the discrepancy is removed if this assumption is dropped and the emission in each line is calculated separately before taking the ratio. These two calculations do not yield the same result because there is a high temperature tail in the ionization curve of the lithium-like ions which results in the transitions of higher excitation energy, the $2 l-3 l^{\prime}$ transitions, being enhanced relative to the $2 s-2 p$ transition. The unsatisfactory agreement between theory and observation could have important implications for current theories of the ionization equilibrium, but, before definite conclusions can be reached, further observations of the same type need to be made and remaining uncertainties in the cross-sections to be resolved.

\section{References}

Flower, D. R.: 1971, J. Phys. B 4, 697 .

Heroux, L. and Cohen, M.: 1971, Phil. Trans. Roy. Soc. London A270, 99.

Jordan, C. : 1969, Monthly Notices Roy. Astron. Soc. 142, 501.

\section{DISCUSSION}

H. Nussbaumer: It is reasonable to doubt the accuracy of atomic data but what about the observed intensities? We are at the mercy of the observers and it might be interesting to hear them attack each other's accuracies.

L. Heroux: The accuracies of the experimental intensity ratio should be approximately $+30 \%$. The experimental ratios discussed by Flower were obtained from a spectrometer designed specifically to carry out these ratio measurements for the lithium-like ions. The spectrometer for the long wavelength resonance lines was calibrated with respect to a tungsten cathode which in turn had been calibrated against an ionization chamber. From the short wavelength lines from the $3 s, 3 p$ and $3 d$ levels, a Geiger-Muller counter was used to calibrate the spectrometer. The chief source of error in the ratio measurement is the error in the photometric calibration of the spectrometer. In comparison to this 
source of error, the statistical errors in counting and errrors due to absorption of the lines by the Earth's atmosphere are small, although small corrections for atmospheric absorption of the resonance lines are necessary. An absolute photometric calibration of the spectrometer was made at several wavelengths over the complete wavelength range 45 to $1236 \AA$.

S. R. Pottasch: What would be the accuracy of the ratio if observations by different observers were combined, instead of a specially designed experiment?

L. Heroux: Different groups have measured absolute intensities photoelectrically in the EUV and usually (except for particular lines) the measurements agree fairly well. To my knowledge intensity ratios of those lines have not been measured in the same rocket experiment by other experimenters and therefore there is no way to compare the AFRCL results with the results of others. 